Nepal Agric. Res. J. Vol. 9, 2009

\title{
PARTICIPATORY CROP IMPROVEMENT OF NEPALESE FingeRMILLET Cultivars
}

\author{
SHARAD BAJRACHARYA ${ }^{1}$, RAM C. PRASAD ${ }^{2}$ AND SHIVA K. BUDHATHOKI ${ }^{2}$ \\ ${ }^{1}$ AgRONOMY DIVISION, NARC, KHUMALTAR, LALITPUR, NEPAL \\ ${ }^{2}$ Hill Crops Research Program, NARC, Kabre, DolaKha, NePAl
}

\begin{abstract}
A FIELD STUDY WAS UNDERTAKEN ON NEPALESE FINGERMILlET GENOTYPeS WITH THE PARTICIPATION OF THE LOCAL COMMUNITY AT PIPALTAR OF NUWAKOT DISTRICT DURING 2003 AND 2004. THE STUDY INCLUDED A) IDENTIFICATION OF SUPERIOR FINGERMILLET CULTIVARS THROUGH DIVERSITY BLOCK MANAGEMENT AND MOTHER SET TRIAL, B) SEED PRODUCTION OF PROMISING LINES, C) FARMERS' FIELD VERIFICATION TRIAL (DIAMOND TRIAL) AND D) CHARACTERIZATION AND DOCUMENTATION OF FINGERMILLET GENOTYPES. RESULTS REVEALED THAT MUdKE, CHAURE AND JALBIRE ARE HIGH YIELDING PROMISING GENOTYPES SUITED TO THAT AREA. FROM HOUSEHOLD SURVEYS CARRIED OUT AMONG 46 SAMPLES (40\%), 80 HH haVe ReVEaled that MudKe, Chaure and Seto Kodo are PREFERRED Fingermillet CUlTIVARS. ABOUt 70\% GROW MUdKe, 46\% GROW ChaURE WHILE 2\% GROW SETO KODO. THE PROGRAMME HAS SUCCESSFULlY EXPLORED THE POTENTIAL OF LOCAL FINGERMILLET CULTIVARS BY THERE EVALUATION AND UTILIZED THEM THROUGH VALUE ADDED PRODUCT DEVELOPMENT AND MARKET PROMOTION.
\end{abstract}

KEY WORDS: CHARACTERIZATION, CULTIVAR, FINGERMILLET, VARIETAL EVALUATION

\section{INTRODUCTION}

Fingermillet (Eleusine coracana Gaertn.), a crop of many poor and subsistent people in the hills of Nepal, is the fourth most important cereal crop in the country. It is mostly grown under a maize/millet cropping system. Its area under cultivation is 2,59,130 ha with the production of $2,82,860 \mathrm{mt}$. (CBS 2061). The national average yield is $1.09 \mathrm{mt} \mathrm{ha}^{-1}$. Even though, the crop is important for the subsistence of rural farming household, its potential has not been fully realized, thus considered neglected and under-utilized in the national perspective. In a food policy review (2001) by International Food Policy Research Institute, it stated that throughout the developing world, poor people subsist on diets consisting of staple foods such as rice or maize and little else. The lack of diversity in the foods they eat often leads to micronutrient deficiencies. This dilemma can be very much true in our country case because people in rural and inaccessible areas have less choice of food items either due to scarcity or poverty. Fingermillet to some extent can mitigate this problem in rural community.

After the initiation of the project, "Enhancing the contribution of Nutritious but Neglected Crops to Food Security and to Increase income of the Rural Poor: Nepal Component of Fingermillet", during 2002 in Hill Crop Research Program, Kabre, diversified studies were carried out in fingermillet crop. The objectives of the project were to conserve and utilize fingermillet genetic resource through development-oriented research and to tackle major causes of it's under use by reviving the cultivation of nutritious but neglected crop. 


\section{MATERIALS AND METHODS}

Five different activities were carried out under varietal evaluation and production management. The activities were diversity block management, mother set trial, characterization of local fingermillet genotypes and lines, seed production and farmers' field verification trial (Diamond trial).

Eighteen local fingermillet cultivars collected from Nuwakot, Kaski and Dolakha were sown in diversity block management for evaluating performance of the landraces. They were sown in rodrow with the spacing of $10 \mathrm{~cm}$ between rows. Plot size was $2 \mathrm{~m}^{2}$. Chemical Fertilizer was applied at the rate of 30:30:30 NPK kg ha ${ }^{-1}$ and FYM 5 ton ha ${ }^{-1}$. In the mother set trial, six fingermillet cultivars were grown in the farmers' fields for selecting farmers' preferred cultivars. The trial was conducted in complete randomized block design with four replications. The plot size was $6 \mathrm{~m}^{2}$ with the spacing of $10 \mathrm{~cm}$ between rows. Fertilizers and FYM were applied at the rate of 30:30:30 NPK $\mathrm{kg} \mathrm{ha}^{-1}$ and 5 ton ha ${ }^{-1}$, respectively. Likewise, 243 fingermillet cultivars and lines were evaluated at Kabre farm. Seed production of the selected fingermillet cultivars like Kabre kodo-1, GPU-25 and Mudke were carried out and diamond trial (Table 1) was conducted in the farmers' fields.

Table 1. Treatment details of Diamond Trial carried out at Pipaltar during 2003 and 2004

\begin{tabular}{|c|c|c|c|c|}
\hline & $\begin{array}{c}\text { Improved } \\
\text { varieties/improved } \\
\text { practice }\end{array}$ & $\begin{array}{l}\text { Improved varieties/Local } \\
\text { practice }\end{array}$ & $\begin{array}{c}\text { Local } \\
\text { varieties/Improved } \\
\text { practice }\end{array}$ & $\begin{array}{l}\text { Local varieties/Local } \\
\text { practice }\end{array}$ \\
\hline 1 & $\begin{array}{l}\text { Improved fingermillet } \\
\text { variety Kabre kodo-1 }\end{array}$ & $\begin{array}{l}\text { Improved fingermillet } \\
\text { variety Kabre kodo-1 }\end{array}$ & \multirow{2}{*}{$\begin{array}{l}\text { Farmer local Mudke } \\
\text { cultivar } \\
\text { 30:30:30 NPK kg } \\
\text { ha }^{-1}\end{array}$} & $\begin{array}{l}\text { Farmer local Mudke } \\
\text { cultivar }\end{array}$ \\
\hline 2 & 30:30:30 NPK kg ha-1 & Urea (small amount) & & Urea (small amount) \\
\hline 3 & $\begin{array}{l}\text { Spacing between rows } \\
10 \mathrm{~cm}\end{array}$ & No line maintenance & $\begin{array}{l}\text { Spacing between } \\
\text { rows } 10 \mathrm{~cm}\end{array}$ & No line maintenance \\
\hline
\end{tabular}

\section{RESULTS AND DISCUSSION}

\section{Diversity block management and mother set trial}

Various qualitative and quantitative traits of different fingermillet genotypes were evaluated in diversity block and mother set trial during 2003 and 2004. The results are presented in Tables 2 and 3.

Table 2. Summery of diversity block management at Pipaltar, Nuwakot during 2003 and 2004

\begin{tabular}{|c|c|c|c|c|c|c|c|}
\hline SN & Cultivars & $\begin{array}{c}\text { Days to } \\
75 \% \\
\text { maturity** }\end{array}$ & $\begin{array}{c}\text { Plant } \\
\text { height, } \\
\mathrm{cm}\end{array}$ & $\begin{array}{c}\text { Plant } \\
\text { stand } / \mathrm{m}^{2} \\
* *\end{array}$ & $\begin{array}{c}\text { No. of } \\
\text { fingers/head }\end{array}$ & $\begin{array}{l}\text { Grain } \\
\text { yield, } \\
\text { kg ha }^{-1}\end{array}$ & Remarks \\
\hline 1 & Seto Kodo & 128 & 99.7 & 72 & 6 & 1248 & \\
\hline 2 & Seto Dalle & 123 & 115.3 & 74 & 8 & 1570 & Good for bread \\
\hline 3 & Kalo Dalle & 126 & 114.2 & 96 & 7 & 1552 & \\
\hline 4 & Kalo Jhyape & 120 & 100.3 & 86 & 7 & 1792 & \\
\hline 5 & Mudke Kodo & 126 & 111.8 & 88 & 7 & 1886 & First preference \\
\hline 6 & Paheli Mudke & 116 & 108.2 & 82 & 8 & 1860 & \\
\hline 7 & Chaure Kodo & 123 & 104.1 & 88 & 8 & 1502 & \\
\hline 8 & Paheli Kodo & 129 & 105.0 & 64 & 7 & 1336 & \\
\hline 9 & Kukurkane & 123 & 102.0 & 58 & 7 & 1530 & \\
\hline 10 & Chaure Kodo & 123 & 106.0 & 88 & 7 & 2040 & \\
\hline 11 & Chitwane Loc. & 123 & 102.8 & 102 & 8 & 1924 & \\
\hline 12 & Jalbire & 129 & 104.5 & 82 & 8 & 1896 & \\
\hline
\end{tabular}




\begin{tabular}{llcccccc}
\hline SN & Cultivars & $\begin{array}{c}\text { Days to } \\
75 \% \\
\text { maturity** }\end{array}$ & $\begin{array}{c}\text { Plant } \\
\text { height, } \\
\mathrm{cm}\end{array}$ & $\begin{array}{c}\text { Plant } \\
\text { stand } / \mathrm{m}^{2} \\
* *\end{array}$ & $\begin{array}{c}\text { No. of } \\
\text { fingers/head }\end{array}$ & $\begin{array}{c}\text { Grain } \\
\text { yield, } \\
\mathrm{kg} \mathrm{ha}^{-1}\end{array}$ & Remarks \\
\hline 13 & Seto Jhyape & 128 & 109.3 & 100 & 7 & 1772 & \\
14 & Kabre Kodo-1 & 121 & 106.9 & 76 & 6 & 1447 & \\
15 & Paheli* & - & 111.9 & - & 8 & 1136 \\
16 & Dalle & 124 & 110.7 & 62 & 7 & 1202 & \\
17 & Farmer's Local & 125 & 117.0 & 96 & 8 & 1541 & \\
(Mudke) & Mean & 124 & 107.6 & 82 & 7 & 1602 \\
& Maximum & 129 & 117.0 & 102 & 8 & 2040 \\
& Minimum & 116 & 99.7 & 58 & 6 & 1136 & \\
& SD & 3.5 & 5.3 & 13.5 & 0.69 & 275.4 & \\
\hline
\end{tabular}

* and ** included in the year 2004 only.

Table 3. Summery of mother set at Pipaltar, Nuwakot during 2003 and 2004

\begin{tabular}{clccccc}
\hline SN & Cultivars & $\begin{array}{c}\text { Days to 75\% } \\
\text { maturity }\end{array}$ & $\begin{array}{c}\text { Plant } \\
\text { height, cm }\end{array}$ & $\begin{array}{c}\text { Plant } \\
\text { stand } / \mathrm{m}^{2}\end{array}$ & $\begin{array}{c}\text { No. of } \\
\text { fingers/head }\end{array}$ & $\begin{array}{c}\text { Grain yield, kg } \\
\text { ha }^{-1}\end{array}$ \\
\hline 1 & Kabre Kodo-1 & 135.2 & 117.4 & 84.3 & 6 & 2588.9 \\
2 & GPU-25 & 133.8 & 99.0 & 90.7 & 6 & 2786.1 \\
3 & GE-5177 & 137.2 & 111.7 & 92.2 & 7 & 1911.1 \\
4 & GE-0122 & 132.5 & 117.1 & 92.2 & 6 & 1844.4 \\
5 & Acc\#523-1 & 135.3 & 113.0 & 89.8 & 6 & 2077.8 \\
6 & Mudke Kodo & 133.8 & 110.8 & 97.3 & 8 & 2755.6 \\
\hline & Mean & 134.6 & 111.1 & 91.1 & 6 & 2327.3 \\
& CV, \% & 3.59 & 10.58 & 17.55 & 13.3 & 58.8 \\
& F-test & $\mathrm{ns}$ & $\mathrm{ns}$ & $\mathrm{ns}$ & $* *$ & $\mathrm{~ns}$ \\
& LSD & 5.7 & - & 17.55 & 0.8 & 1360 \\
\hline
\end{tabular}

\section{Seed production of promising cultivars}

With an aim to supply quality seed and effort to conserve superior fingermillet local genotypes insitu, seed production of elite fingermillet cultivars were carried out (Table 4).

Table 4. Estimated yield of fingermillet from seed production activities at Pipaltar during 2003 and 2004

\begin{tabular}{|c|c|c|c|c|c|}
\hline \multirow{2}{*}{$\mathrm{SN}$} & \multirow{2}{*}{ Cultivars } & \multicolumn{2}{|c|}{ Year 2003} & \multicolumn{2}{|c|}{ Year 2004} \\
\hline & & Farmers & Yield, $\mathrm{kg} \mathrm{ha}^{-1}$ & Farmers & Yield, $\mathrm{kg} \mathrm{ha}^{-1}$ \\
\hline 1 & Kabre Kodo-1 & Birman Kumal & 2420 & Bishwo Kumal & 2928 \\
\hline 2 & GPU- 25 & Bishwo Kumal & 3300 & Bishwo Kumal & 3725 \\
\hline 3 & Mudke & Resham Kumal & 2530 & - & - \\
\hline
\end{tabular}

\section{Farmer's field verification trial (Diamond trial)}

This verification trial demonstrated the performance of improved method of cultivation over farmers' practice. Local cultivar, Mudke, with improved practice gave the highest yield followed by improved variety Kabre Kodo- 1. 
Table 5. Results from the Diamond trial conducted at Pipaltar during 2004

\begin{tabular}{|c|c|c|c|c|c|c|c|}
\hline $\begin{array}{l}\text { Trt } \\
\text { No }\end{array}$ & Treatment & $\begin{array}{l}\text { Grain } \\
\text { yield, } \\
\mathrm{kg}_{1} \mathrm{ha}^{-}\end{array}$ & $\begin{array}{l}\text { Plant } \\
\text { heigh } \\
\mathrm{t}, \mathrm{cm}\end{array}$ & $\begin{array}{c}\text { Maturit } \\
\text { y days, } \\
75 \%\end{array}$ & $\begin{array}{c}\text { No. of } \\
\text { plants } / \mathrm{m}^{2}\end{array}$ & $\begin{array}{c}\text { No. of } \\
\text { head } / \mathrm{m}^{2}\end{array}$ & $\begin{array}{c}\text { No. of } \\
\text { fingers/hea } \\
d\end{array}$ \\
\hline 1 & $\begin{array}{l}\text { Imp. cv./Imp. } \\
\text { Practice }\end{array}$ & 2950 & 111 & 131 & 100 & 103 & 7 \\
\hline 2 & $\begin{array}{l}\text { Imp. cv../Local } \\
\text { practice }\end{array}$ & 2650 & 106 & 131 & 108 & 110 & 6 \\
\hline 3 & $\begin{array}{l}\text { Local cv./Imp. } \\
\text { practice }\end{array}$ & 3575 & 97 & 125 & 112 & 114 & 6 \\
\hline \multirow[t]{2}{*}{4} & $\begin{array}{l}\text { Local cv./Local } \\
\text { practice }\end{array}$ & 2850 & 96 & 125 & 89 & 95 & 7 \\
\hline & Mean & $\begin{array}{c}3006 . \\
2\end{array}$ & 102.5 & 128 & 102.25 & 105.5 & 6.5 \\
\hline
\end{tabular}

Characterization and documentation of fingermillet genotypes

Two hundred forty-three fingermillet genotypes/ lines were evaluated at Kabre, Dolakha during 2004 (Table 6 and Table 7). Variations in both the qualitative and quantitative characteristics were observed among the tested genotypes that could be utilized in the future breeding programmes.

\section{Table 6. Few Qualitative characteristics of 243 fingermillet lines assessed at Kabre during 2004}

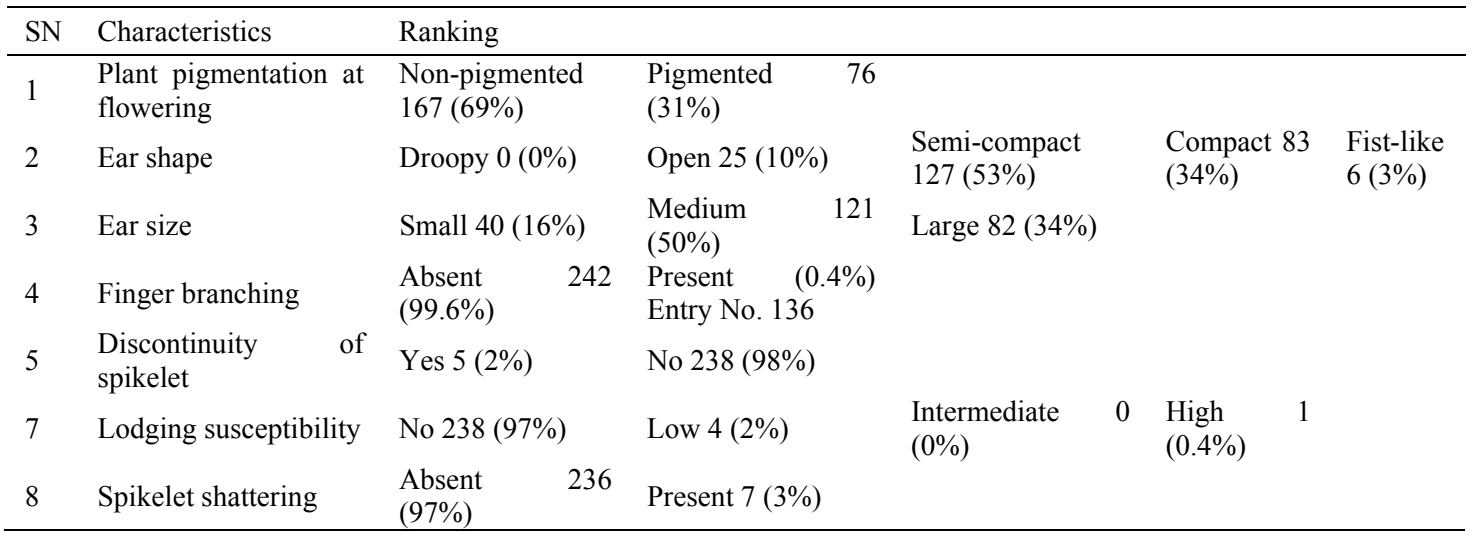

Table 7. Quantitative characteristics of 243 fingermillet genotypes/lines assessed at Kabre during 2004

\begin{tabular}{llrrrrr}
\hline SN & \multicolumn{1}{c}{ Traits } & Minimum & Maximum & Mean & CV, \% & SE of mean \\
\hline 1 & Plant height, cm & 27 & 107 & 80 & 17 & 0.87 \\
2 & Flag L/W ratio & 8.7 & 3.4 & - & - & - \\
3 & Culm branching & 1 & 3 & 1.3 & 36.3 & 0.03 \\
4 & Culm thickness, mm & 5 & 15 & 8 & 16.1 & 0.07 \\
5 & Productive tillers & 1 & 3 & 1.3 & 36.3 & 0.03 \\
6 & Finger L/W ratio & 6.4 & 8 & - & - & - \\
7 & Disease scoring & 1 & 7 & 1.3 & 61 & 0.05 \\
\hline
\end{tabular}


Fingermillet contributed $22 \%$ on the total food sharing in Tallo Pipaltar (Khadka et al 2005). To increase the value of any local crop diversities, experts working in this area must understand the different values that the local crops hold for farmers as well as the ways in which changing social and technological conditions will affect those values. The project has been successful in collecting resource of information on the local fingermillet cultivars in respect to social setting. Large numbers of Nepalese fingermillet cultivars have been assessed in terms of their yield performance as well as agro morphological characteristics, and superior genotypes like Mudke Kodo, GPU-25 and Chaure Kodo have been identified as promising fingermillet cultivars. Moreover so, the project has helped in the utilization, promotion and in the conservation of the countries rich fingermillet resources.

\section{ACKNOWLEDGEMENTS}

The authors wish to thank Mr R Dahal for his dedicated effort and support in conducting the experiments. The support received from the whole HCRP team is also highly appreciated.

\section{REFERENCES}

CBS. 2061. Statistical information on Nepalese agriculture 2060. Central Bureau of Statistics. Agricultural Statistics Division, HMG/Nepal. Singha Durbar, Kathmandu, Nepal.

Khadka R, RP Upreti, S Gautam, YN Ghimere, D Gauchan and S Shrestha. 2005. Baseline study on Fingermillet in Pipaltar, Bidur Municipality, Nuwakot District. In: A Survey report on fingermillet of Nuwakot District (Pipaltar and Khanigaun) under IFAD-NUS Project. Nepal Agricultural Research Council. 